Review Article

\title{
A Survey on Microgrid Control Techniques in Islanded Mode
}

\author{
Uthpala N. Ekanayake $(\mathbb{D})$ and Uditha S. Navaratne $\mathbb{D}$ \\ Department of Electrical and Electronic Engineering, University of Peradeniya, 20400 Peradeniya, Sri Lanka
}

Correspondence should be addressed to Uthpala N. Ekanayake; uth7ekanayake@gmail.com

Received 16 June 2020; Revised 21 September 2020; Accepted 28 September 2020; Published 15 October 2020

Academic Editor: Andrea Bonfiglio

Copyright (c) 2020 Uthpala N. Ekanayake and Uditha S. Navaratne. This is an open access article distributed under the Creative Commons Attribution License, which permits unrestricted use, distribution, and reproduction in any medium, provided the original work is properly cited.

Traditional power networks with generation in upstream and consumption in the downstream were controlled with centralized controls like SCADA. However, in order to facilitate the penetration of distributed generation, the concept of microgrid was popularized. A microgrid can operate both in grid-connected and in islanded modes. One of the challenges in the microgrid environment is to provide both voltage control and maintain the system frequency while ensuring the stability of the network. This paper is a literature survey focused on different microgrid control techniques with different levels of communication especially in islanded operation.

\section{Introduction}

With the high penetration levels of renewable in the power network, the traditional centralized network posed many technical issues for maintaining stability and protection. Limited resources and high energy demand have resulted in many governments to set up policies to promote renewable energy. The energy generation which was limited to the top level of the network hierarchy has now expanded to the distribution level with the increasing use of solar PV and other renewable sources like wind. The control and monitoring once seen in the generation and transmission have now become a must, even at the distribution. The concept of microgrids has facilitated the integration of renewable resources at this level. A microgrid is a small-scale distribution network containing a set of distributed generations (DGs) designed to supply electrical power to a local area (LA), connected to a large network (host grid) as a single controllable entity [1]. Microgrids became popular because of their ability to work in isolation.

A microgrid operation can be in two modes. When the microgrid fulfills its energy demand by the main grid, it is called grid-connected mode and when demand is supplied from its own local generation, it is called islanded mode. In grid-connected mode, the main objective of a controller is to provide energy management, while in islanded mode, the objective is to control both its frequency and voltage, while supporting the energy demand. If there are no synchronous machines to balance demand and supply for island operation, the inverter is responsible for providing these controls especially the frequency control [2-4]. PQ (Active Power and Reactive Power) control is the control strategy used in the grid-connected mode for power balance and it switches to $\mathrm{V} / f$ (voltage frequency control), when the microgrid is in islanded mode [2]. The most common approaches in grid control architecture are centralized control and decentralized control architectures. The centralized control approach is widely used in traditional power networks mainly at the generation level with Supervisory Control Data Acquisition (SCADA) systems [5]. Here, the control operation is planned beforehand and is implemented. With distributed generation, decentralized control architectures became popular.

\section{Centralized Control}

A central controller is used with distributed sensors, actuators, local controllers, and supporting communication network. Data collected from the distributed sensors are sent to the centralized control via a communication network. 
Once the central controller gathers data, it calculates the control variable for each control equipment and sends them back to the local controllers $[4,5]$. Local controls never perform decision-making and all the decisions are made at the central controller. This is illustrated in Figure 1. Here, the abbreviation LA corresponds to the local area controller and $\mathrm{CC}$ to the central controller.

\section{Decentralized Control}

In a decentralized control approach, the distribution system is divided into small networks called microgrids. A microgrid can have a few local area (LA) networks and its own controller. In a fully decentralized approach, each unit (DER) in a microgrid is controlled by its local controller (LC) [4-6]. An LC supervises a set of LAs that require no communication between LAs. When the LCs communicate to find a common solution for the overall control problem, the control architecture is called distributed.

Islanded microgrid operation is challenging due to the intermittent nature of renewable energy generation. They create uncertainties in maintaining a stable voltage and frequency output. Hence, this shows the requirement of an accurate load forecasting and load management system with a decentralized nature. However, a fully decentralized approach is not possible as it requires strong coordination between the LCs.

\section{Distributed Control}

Decentralized and distributed control operates on the same principle except that distributed architecture has communication with local networks (LAs) and LCs. A distributed control scheme is illustrated in Figure 2.

Distributed controls assign control tasks to different DGs and their interaction. These control tasks are based on different time frame operations and they constitute a control hierarchy. In a network, when the number of units increases, distributed control is challenging unless a control hierarchy is established. This approach is used for the coordination of each unit. This will be discussed further in the next section. The distributed techniques aim to address an underlying optimization problem in a distributed manner with limited communication [7-9].

Distributed control strategies include model predictive control-based techniques, consensus-based techniques, and agent-based techniques, which will be discussed in later sections. The recent interest in research of distributed control strategies shows microgrid island operation and control together with preserving privacy and protecting the system from cyberattacks [7].

\section{Hierarchical Control}

The hierarchical control system has two concepts, namely, multilayer and multilevel. In a multilayer concept, the control is split into layers and each acts at different time intervals. A hierarchical control scheme consists of three levels of control: primary, secondary, and tertiary $[6,7]$. The controller has different control layers to coordinate the control process. While the upper layers manage the objectives globally by providing control set points, the lower layers perform the controlling. In a multilevel concept, control is introduced into local controllers (LCs) and their action is coordinated by an additional unit MCC (Microgrid Central Control) with the Main Grid Central Controller. A hierarchical control-based control architecture is illustrated in Figure 3 [6].

The control levels in the hierarchy are illustrated in Figure 4 [10]. It is necessary to ensure the control from one level to the lower levels and the communication bandwidth of the hierarchical control is decreased with an increase in control level.

The main purpose of the primary level controller is to provide direct control to the electronic converters to maintain voltage and frequency; the secondary level is responsible to establish coordination among the neighboring local controllers and mitigate voltage/frequency deviations caused at the primary control level. In the third level and the highest level, the controller takes all the uncertainties of the power network to determine the optimal power flow ensuring demand/generation balance $[10,11]$.

5.1. Primary Control. Primary control is the first level in the hierarchy dealing with the local controllers. They require a fast response with minimum or no communication to control problems in the power network. The primary purpose is islanding detection and voltage/frequency control [6-11]. Primary control can be categorized into master/slave control and distributed control which requires communication links to be established and other categories of droop controls which require no communication. Since master/ slave control requires communication channels with higher bandwidths and it reduces the reliability in case of failure of a single point of the network, the widely adapted method is droop control [12]. Primary control is performed by Voltage Source Inverter (VSI) controllers.

The inverter that interfaces the DERs to the grid works in two ways. It works in Power Control Mode (PCM) when operating in grid-connected mode and Voltage Control Mode (VCM) when operating in islanded mode [4]. VCM control is used to regulate the output of the VSI where droop characteristics are used to control voltage and frequency. The droop controls are described using equations (1) and (2) as follows:

$$
\begin{gathered}
\omega=\omega_{0}-K p * P g, \\
V=V_{0}-K q * Q g,
\end{gathered}
$$

where $\omega$ and $V$ are the new steady-state frequency and voltage values, respectively, $\omega_{0}$ and $V_{0}$ are the base frequency and voltage values fixed when operating in gridconnected mode, $K p$ and $K q$ are the active power and reactive power static droop gains, and $P g$ and $Q g$ are the threephase injected active power and reactive power. 


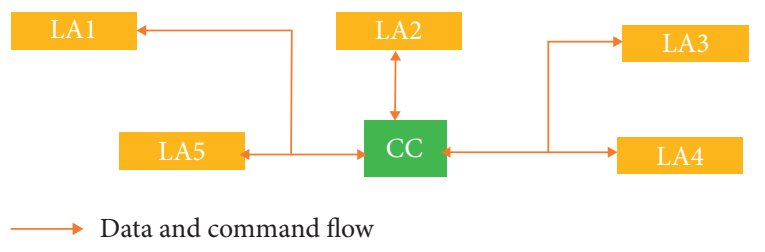

FIgURE 1: Centralized control architecture. All local area networks or microgrids in the main network are controlled by a centralized controller.

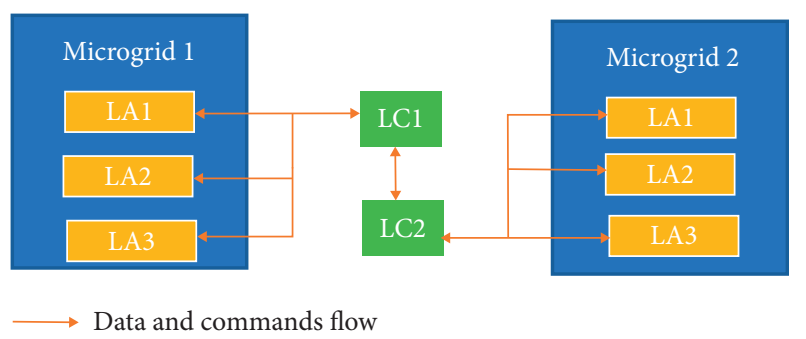

FIGURE 2: Distributed control architecture. A controller is assigned to each microgrid that can communicate with each other.

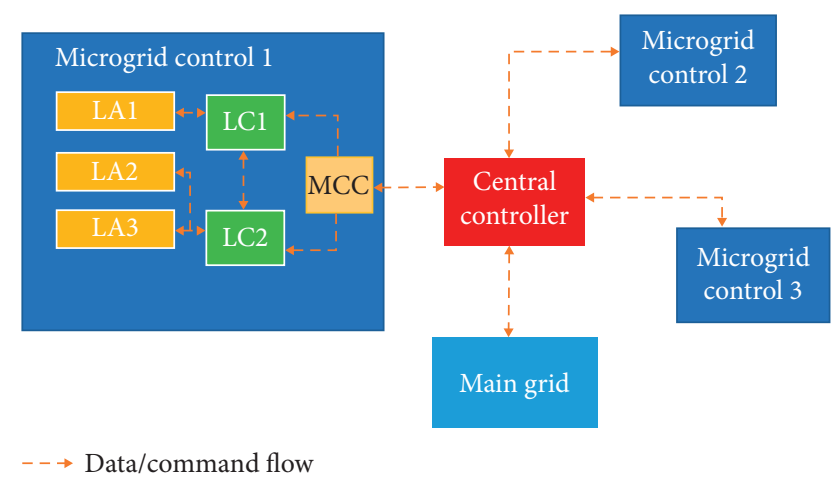

Figure 3: Hierarchical control architecture. MCC coordinates the actions of LCs with a main grid.

Droop control realizes active power with droop characteristics to improve the frequency and reactive power to improve the output voltage of the DG units. However, there are disadvantages involved in droop controls. They are poor in voltage regulation and have high voltage distortion, a probability of a loss of synchronism when resynchronizing with the main grid [2] and most importantly poor reactive power sharing among the DERs. Droop control guarantees the stability of frequency and correct active power sharing. However, it produces errors in reactive power sharing. This is highlighted through grid parameters relevant to the regulation problem in [12]. If $Q_{i}^{\prime}$ is the reactive power delivered to the Point of Common Coupling (PCC) and V is the voltage at PCC, then

$$
Q_{i}^{\prime}=\left(\frac{V i-V}{X i}\right) V .
$$

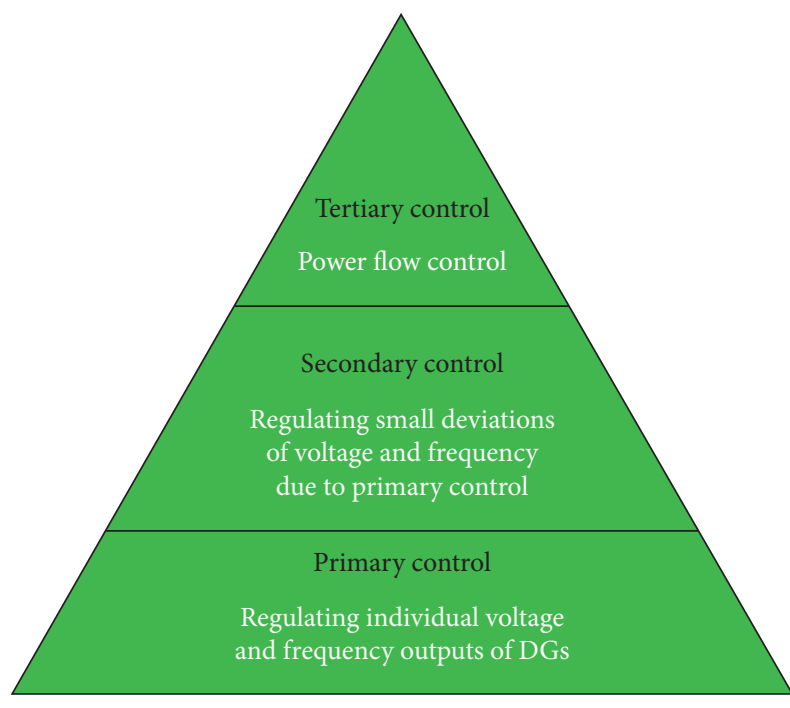

Figure 4: Representation of control hierarchy [10].

According to equation (3), the connection impedance to the PCC affects the correctness of reactive power sharing. Apart from this, the reactive power setpoint in droop controls from an energy management system (EMS) and the increase in load demand also affects the reactive power sharing.

Nondroop methods such as described in [13] use powersharing methods. A decentralized servomechanism controller at each DG unit adjusts its voltage independently. Power sharing is achieved using an internal oscillator that extracts the phase angle and transmits it to DGs. However, this type of method is difficult to implement in larger systems.

Recent developments include the modeling of the inverter as a virtual generator. Its goal is to mimic the conventional synchronous machine dynamics to improve voltage and frequency stability $[14,15]$.

Reference [14] describes a Synchronverter that mimics the behavior of a synchronous generator employed with modifications to the software without changing the hardware. These modifications can include virtually controlling the field current, so that it delivers a better way of computing reactive power, increasing the size of filter inductors to make the inverter more robust towards small voltage violations, virtually changing the nominal active mechanical torque, and introducing virtual capacitors to eliminate the influence from DC voltages.

Reference [15] describes a comprehensive inverterBESS-based primary control. The control of this system can regulate frequency and voltage in microgrid islands in VCM mode, independently of the number of parallel operations of other DERs, with the adaptation of a virtual generator. This technique includes an automatic voltage regulator and a rotor flux model which models the reactive power/voltage magnitude similar to that of a synchronous generator through machine flux variation as in equation (4) and an inertia model and a frequency governor that makes the system similar to a synchronous generator active power/ 
frequency dynamics through virtual mechanical dynamic as in equation (5):

$$
\begin{aligned}
\frac{\mathrm{d} v_{\text {ref }}}{\mathrm{d} t} & =k_{i}\left(Q_{\mathrm{AvR}}-Q\right), \\
\frac{\mathrm{d} \omega_{\text {ref }}}{\mathrm{d} t} & =K\left(P_{\text {gov }}-P-k_{d} \omega_{\text {ref }}\right) .
\end{aligned}
$$

$v_{\text {ref }}$ is the initial voltage variation, $k_{i}$ is rotor flux model gain which is modeled as an integrator, and $Q_{\mathrm{AVR}}$ and $Q$ are reactive power at the output of the AVR and the inverter, respectively. $\omega_{\text {ref }}$ is the rotational speed of the virtual generator, $K$ is inertial model gain, $k_{d}$ is the damping effect, and $P_{\text {gov }}$ and $P$ are the active power at the output of the governor and the inverter, respectively.

Secondary control level is used to mitigate the problems aroused in the primary control.

5.2. Secondary Control. Secondary control is required in both grid-connected and in islanded modes. However, the latter is challenging due to the uncertainties of generation in DGs and the update rate of load dispatch commands $[3,4]$. The main objective at this level hence includes finding the optimal dispatch of all DERs and restoring the voltage and frequency deviations produced at the primary control. Realtime optimization is required at this level for economic dispatch and unit commitment.

Secondary control is the highest hierarchical level in islanded microgrids $[3,4]$. This control operates in a slower time frame than the primary control reducing the communication bandwidth used by the microgrid variables. Hence, it allows the performance of more complex calculations. The secondary control level can be centralized or distributed. In centralized secondary control, MCC manages power flow and provides reference values for primary control. After gathering data, it determines the deviations and decides the secondary control action [16]. However, this approach is highly dependent on communication and is more expensive and unreliable. Recent developments show distributed control approaches at this level to reduce communication.

In literature, [17] presents a distributed secondary control (DSC) scheme for inverters in a network with uncertain communication links. The discrete-time DSC controllers use an iterative learning mechanism that enables the DERs in the microgrid to achieve voltage/frequency restoration and active power sharing. Here, the secondary control inputs are updated at the end of an iteration round; thus, DERs only need to share data with its neighbors in low bandwidth.

The secondary controllers are not necessary to be frequently operated. Hence, [18] suggests an event-triggered approach. The controller is updated only when a local measurement error exceeds a certain boundary, thereby limiting the communication. Because of the existence of communication noise among DGs, [19] describes a fully distributed noise-resilient secondary voltage control approach to select a proper set of control inputs for voltage and frequency restoration.
An optimal operation is sought in secondary control, also referred to as the energy management of a microgrid, through the implementation of multiagent systems where individual DER units are controlled by local agents.

5.3. Tertiary Control. Tertiary control is the highest and the lowest level of control for grid-connected microgrids operating in hierarchical control schemes. It sets long-term set points to operate for DER units. Moreover, it is responsible for managing multiple microgrids in a host network [2-4]. This control level applies to the operation and managing power flow between the microgrid and the main grid. In islanded mode, tertiary control gets disabled. Hence, it is not discussed in detail in this survey.

\section{Agent-Based Techniques for Distributed Control}

Energy management systems with intelligent decisions and adaptive feedback have complex behaviors. Hence, multiagent applications became popular in this domain. A multiagent system decomposes a complex problem into subproblems. The subproblem decisions are then taken by an agent attempting to achieve a certain objective. An agent could be any DER, a load, a transformer, or any other device or any entity in the network [20-22]. In islanded operation, a multiagent system will control DER units based on the information exchanged by the agents through a local controller. They perform negotiations and energy trading to meet load demands [20-22]. The control and communication architecture of these agents could be in centralized, distributed, or hierarchical control. While the agents in local areas are responsible for communicating with each other to solve the control issues locally, there can be coordination with a communicative agent among them. This is illustrated in Figure 5.

Centralized agent architecture consists of agents managed by a single controller in a master-slave relationship [22]. Distributed agent architecture consists of several agents allowed to discover global information through communication with their neighbors. Hierarchical agent consists of different layers of agents whose communication is achieved via a layered architecture. The information flow is from lower layers to higher layers, whereas the control flow is from higher layers to lower layers. Besides information flow in layers, different agents in the same layer may also be able to communicate with each other.

6.1. Centralized Agent Architecture. Here, a collection of nonintelligent agents is managed by a central controller in a master-slave approach. As described in [22], the agents are categorized into two classes: reactive and cognitive. The central controller is a cognitive agent with intelligence responsible for communicating with nonautonomous reactive agents to establish the desired control plan like managing and disconnecting noncritical loads. A diagram in Figure 6 describes these two classes of agents. 


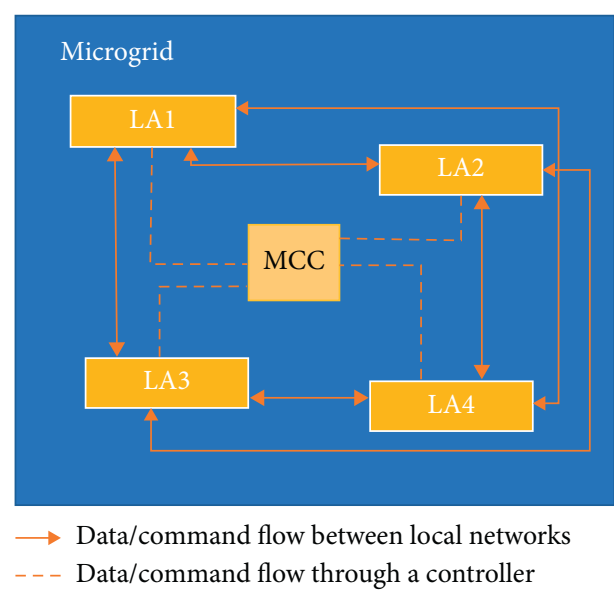

FIGURE 5: Distributed control with agents. This diagram illustrates the data and command flow between the local areas through agents as well as how the commands are centralized using a microgrid controller.

6.2. Distributed Agent Architecture. Here, a collection of agents is handled by a single layer control structure instead of hierarchical layers. The agents are intelligent and autonomous and responsible for managing a local area in the network. These agents can discover global information through communication and coordination with their neighboring agents [22].

6.3. Hierarchical Agent Architecture. In this architecture, agents have different authorities over the control actions. While some agents have more authority to sense and manage over the others. Typically, the upper layer/level agents are responsible for handling a large amount of data and maintaining the communication schedules for demand/ generation balance. The middle layer/level agents are responsible for making decisions, switching between grid connection and islanded modes, minimizing the energy losses, and directing the necessary actions for neighboring agents, in identifying faults and restoring power. The lower layer/level agents are responsible for interacting with sensors in the microgrid. Sensors are responsible for sensing and controlling the grid breakers, DGs, battery energy storage devices (BESS), and controllable loads [22].

\section{Peer-to-Peer Communication}

Peer-to-peer communication is considered a technique that can be used in multiagent systems. Each agent requires a measurement of its own and its nearest neighbor and requires a very low bandwidth for this communication [16]. Here, all the agents have the same priority and the importance of communicating in a peer-to-peer fashion [6]. Algorithms used for P2P communication between the agents include gossiping and consensus algorithms. Gossip algorithms can be used for voltage and frequency control at the secondary level.

These algorithms aim at sharing data quickly in the network [6]. To facilitate the absence of a central controller,



Figure 6: Centralized multiagent architecture. This diagram shows the two types of agents who are responsible for communicating in control architecture, the same as in Figure 5.

these agents require some sort of intelligence making the microgrid autonomous.

Over the past few years, extensive research has been conducted on multiagent systems (MAS). MAS-based energy management system (EMS) architectures have been widely used for distributed control and demand-side energy management in microgrids. Literature [23] presents a MASdecentralized EMS that employs fuzzy cognitive maps for energy management, [21] presents an outage management system using MAS, and in [24], microgrid local controllers are assigned to some specific agents to interact with each other to achieve a common decision in the restoration of voltage. All these studies demonstrate the beneficial application of MAS in microgrids, where the players/agents were cooperating and various decision-making methods and control strategies were used to attain the optimal microgrid performance using techniques like Particle Swarm Optimization, game theory, and generic algorithms.

\section{Model Predictive Control}

In the future microgrid, a control hierarchy with multiagent will be coordinated by a centralized optimization problem to fulfill a control goal. These control strategies can be categorized to direct control and indirect control. A direct control has a two-way communication for accessing the DERs, then communicating back the control strategy, and getting feedback. On the other hand, indirect control needs only one way to initialize DER control. This is where the model predictive control (MPC) strategies come into play. MPC can predict the next control sequence along with a control action (e.g., variation in output of a DER).

Extending MPC to multiagents involves knowing the subsystem model of a control agent in advance. However, a control agent has no global information but can access only local information. Therefore, predictions are important about the future evolution of external variables of neighbors. However, when the horizon becomes longer, the accuracy can get lost. Research work suggests that this could be mitigated by assuming a constant value over the whole horizon based on a local measurement or obtained from a neighboring agent. It is also possible that assuming predictions over the whole horizon or assuming a model that 


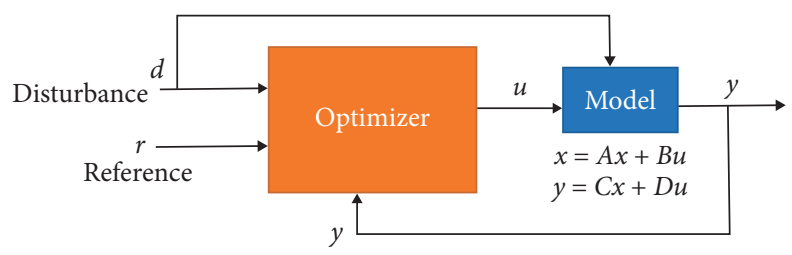

Figure 7: Model predictive control diagram.

predicts the values of the external variables based on the dynamics of neighboring subsystems. [25].

The MPC diagram is illustrated in Figure 7.

The commonly used MPC strategy is called Distributed Model Predictive Control (DMPC). In this discrete time, control methodology, an optimal controller, is developed based on the dynamic system model and forecasting. The MPC control objective is a real-time optimization problem and is repeatedly computed for all control inputs. The optimization problem can be based on minimizing costs or forcing the system to follow predefined control set points based on a prior state estimation. Literature in $[26,27]$ describes different objective functions, based on traditional linear quadratic regulators, economic objectives, and a combination of both.

The MPC scheme has been used to derive linearized models for voltage control in [27]. It predicts the voltage profile in the next time step and adjusts the voltage and reactive power set points accordingly, to achieve a smooth voltage profile in the next step. Equation (6) is the objective function, as given in [27], based on optimizing the generation cost of a microgrid operating in islanded operation:

$$
\min F(U, k)=\sum_{t=k}^{k+N-1}\left(C_{g}(t)+\sum_{t=m} C_{m}(t)\right)
$$

where $C_{g}(t)$ is the generation cost from the main grid proportional to power demand, $C_{m}(t)$ is the cost of the $m^{\text {th }}$ conventional distributed generator in the microgrid, and $U$ is a vector containing control actions in the entire control horizon given by equation (7):

$$
U(t)=\left[u^{T}(k) u^{T}(k+1) \cdots u^{T}(k+N-1)\right]^{T} .
$$

Here, for any single time step, the control signal $U(t)$ includes power outputs of all controllable generators as well as energy storage.

MPC is a type of dynamic control that requires a quick response. This is illustrated in Figure 8 with other control strategies. DMPC and the coordination between the local controllers can be again categorized into distributed and hierarchical communication.

MPC is also used for inverter controls [28, 29] as a primary level control technique. Literature in [28] has used this technique for decentralized primary control of an islanded microgrid (MG) composed only by power converters and without any rotating electrical machine and was tested to an islanded portion of the Smart Polygeneration
Microgrid (SPM) testbed facility at the University of Genoa, Italy.

In [29], the approach focuses on solving optimization problems of MPC with an analytical optimal solution for unconstrained mode and near-optimal solution in a transient state. It gives an output with a fixed switching frequency, increases robustness under system parameter uncertainties and variations, and gives a fast-dynamic response.

\section{Consensus-Based Technique}

In consensus-based techniques, a distributed optimization problem is solved to converge DER operating set points to a single value. In $[30,31]$, consensus-based multiagent schemes are used for load restoration, fault recovery, and frequency control. The literature in [30] describes a distributed voltage secondary control (DVSC) strategy using a dynamic consensus protocol. Here, a voltage correction is provided to droop control units using consensus-based protocol. As shown in Figure 9, to regulate the voltage at a node $i$, the responsible agent has a controller that uses a dynamic consensus estimate given by equation (8):

$$
\dot{V}_{e}(t)=\sum_{j=1}^{N} a_{i j}\left(V_{j}(t)-V_{e}(t)\right)+V_{i} .
$$

Here, $V_{i}$ is the measured voltage at node $i, V_{e}$ is the estimate of the average voltages provided at node $I$, and $V_{j}$ is the voltage estimate received by the neighboring node $j$. The estimated voltage is then compared with a reference microgrid voltage and the error is fed into a PI controller. Since the consensus control is dependent on the degree of connectivity between its agents, different communication topology exists between the DERs. The daisy chain topology, loop communication topology, and star topology are some of these examples.

The principle of defining the consensus method is based on equation (8) and two theories. Here, $a_{i j}$ is the element of the adjacency matrix of the graph and $\mathrm{V}$ is the graph nodes in graph theory. These graph nodes must satisfy the principle of distributed consensus. The first theory says that the graph consists of a spanning tree (a subset of a graph which has all the vertices covered with a minimum possible number of edges) causing a consensus control and all eigenvalues have zero or positive parts. The second theory is that if a graph consists of a spanning tree, all agents' states will converge to the external control signal. These are the fundamentals used in consensus control [32]. 


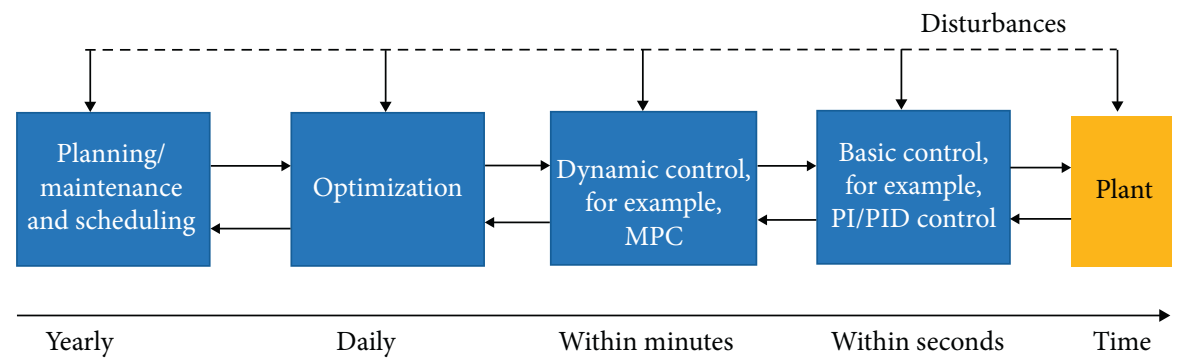

FIgURE 8: A control hierarchy with respect to a timeline. This diagram illustrates when and where MPC which is a dynamic control is placed in a control hierarchy.

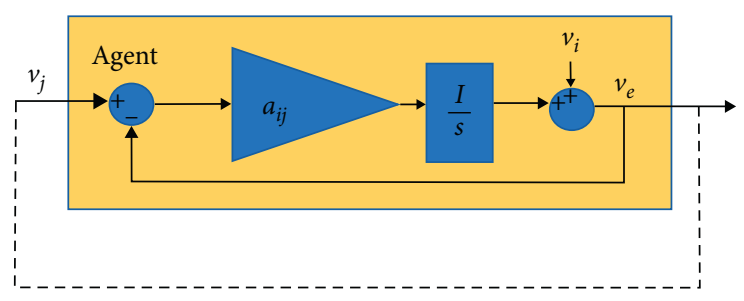

Communication path with neighboring agents

FIgURE 9: Consensus-based control diagram. This diagram shows how consensus-based algorithm is used for voltage regulation.

Distributed generation can be physically placed in widely spanned locations. The consensus-based method can bring these generators to a global agreement of a parameter using only communication among neighbors [31].

\section{Conclusions}

The literature survey describes the techniques used for microgrid control and communication giving relevance to the islanded mode operation. Islanded microgrid controls are responsible for making decisions on maintaining power balance and providing voltage and frequency control. This includes the equilibrium, supply surplus, and the supply shortage. Droop controls can maintain discrepancies in voltage and frequency. However, they still can deviate from their reference values. Recent developments include a nondroop control technique at the primary level where the inverters mimic the conventional synchronous machine dynamics. Additional control levels are introduced as secondary controls in islanded microgrids to mitigate the deviations at the primary level. While the traditional secondary control methods include a centralized structure, the recently developing concepts focus more on distributed control structures. Instead of gathering global information, these controls can use local data collected from neighbors. One of the challenges in distributed control is ensuring that local control actions are consistent with the action of others.

Agent-based techniques are popularized which can use peer-to-peer communication strategies as intelligent agents, attempting to achieve an objective using neighboring data. In a multiagent setting where DMPC has employed, each control agent makes a prediction of its subsystem over a horizon from local data. Due to the constraints of other subsystems, these predictions by their respective agents, communication between the neighboring agents must be accurate enough to improve the decision-making process. However, what information to be communicated, and when to decide on the control action are still some open research in this area. In the case of frequency restoration, the distributed consensus-based control algorithms have demonstrated more accuracy. From all the mentioned algorithms, the consensus-based control used with an agent-based scheme requires the least communication resources and has the fastest convergence time that suits the microgrid secondary control in restoring variations in voltage and frequency during islanded operation.

\section{Data Availability}

The data used to support the findings of this paper are included within the article.

\section{Conflicts of Interest}

The authors declare that there are no conflicts of interest regarding the publication of this paper.

\section{References}

[1] K. V. Vidyanandan and B. Kamath, "Grid integration of renewables: challenges and solutions," in Emerging Energy Scenario in India-Issues, Challenges and Way Forward Neyveli, Tamil Nadu, India, 2018.

[2] O. Palizban and K. Kauhaniemi, Microgrid Control Principles in Island Mode Operation, PowerTech, Grenoble, France, 2013.

[3] K. C. Soni and F. F. Belim, "MicroGrid during grid-connected mode and islanded mode-A review," International Journal of 
Advanced Engineering and Research Development, vol. 20, pp. 2348-4470, 2016.

[4] P. Borazjani, N. I. A. Wahab, H. B. Hizam et al., "A review on microgrid control techniques," IEEE Innovative Smart Grid Technologies-Asia (ISGT ASIA), vol. 2014, pp. 749-753, 2014.

[5] D. E. Olivares, A. Mehrizi-Sani, A. H. Etemadi et al., "Trends in microgrid control," IEEE-PES Task Force on Microgrid Control, vol. 14, 2014.

[6] A. Shreshtha, B. P. Hayes, and R. Bishwokarma, "Peer-to-peer energy trading in micro/mini-grids for local energy communities," A Review and Case Study of the Nepalese Electricity System, vol. 7, no. 1, pp. 131911-131928, 2019.

[7] M. Yazdanian and A. Mehrizi-Sani, "Distributed control techniques in microgrids," IEEE Transactions on Smart Grid, vol. 5, no. 6, pp. 2901-2909, 2014.

[8] H. Almasalma, J. Engels, and G. Deconinck, "Peer-to-peer control of microgrids," in Proceedings of the Young Researchers Symposium, Eindhoven, Netherlands, 2016.

[9] Z. A. Obaid, L. M. Cipcigan, L. Abrahim et al., "Frequency control of future power systems:reviewing and evaluating challenges and new control methods," Journal Modern Power Sytems and Clean Energy, vol. 8, 2019.

[10] N. Yusof1 and Z. Ali, "Review of active synchronization for renewable powered microgrid", International Journal of Engineering \& Technology, vol. 8, no. 17, pp. 14-21, 2019.

[11] L. Meng, Hierarchical control for optimal and distributed operation of microgrid systems, Ph.D. thesis, Aalborg University, Denmark, Europe, 2015.

[12] A. Rosini, M. Minetti, G. B. Denegri, and M. Invernizzi, "Reactive power sharing analysis in islanded AC microgrids," in Proceedings of the 2019 IEEE International Conference on Environment and Electrical Engineering and 2019 IEEE Industrial and Commercial Power Systems Europe (EEEIC/ I\&CPS Europe), IEEE, Genoa, Italy, pp. 1-6, September 2019.

[13] R. Moradi, H. Karimi, and M. Karimi-Ghartemani, "Robust decentralized control for islanded operation of two radially connected DG systems," in Proceedings of the 2010 IEEE International Symposium on Industrial Electronics (ISIE), London, UK, July 2010.

[14] V. Natarajan and G. Weiss, "Synchronverters with better stability due to virtual inductors, virtual capacitors, and antiwindup," IEEE Transactions on Industrial Electronics, vol. 64, no. 7, pp. 5994-6004, 2017.

[15] M. Fusero, A. Tuckey, A. Rosini, P. Serra, R. Procopio, and A. Bonfiglio, "A comprehensive inverter-BESS primary control for AC microgrids," Energies, vol. 12, p. 381, 2019.

[16] P. Singh, P. Paliwal, and A. Arya, "A review on challenges and techniques for secondary control of microgrid"," IOP Conference Series: Materials Science and Engineering, vol. 561, Article ID 012075, 2019.

[17] X. Lu, X. Yu, J. Lai, J. M. Guerrero, and H. Zhou, "Distributed secondary voltage and frequency control for islanded microgrids with uncertain communication links," IEEE Transactions on Industrial Informatics, vol. 13, no. 2, pp. 448-460, 2017.

[18] Y. Wang, T. L. Nguyen, Y. Xu, Z. Li, Q.-T. Tran, and R. Caire, "Cyber-physical design and implementation of distributed event-triggered secondary control in islanded microgrids," IEEE Transactions on Industry Applications, vol. 55, no. 6, pp. 5631-5642, 2019.

[19] N. M. Dehkordi, H. R. Baghaee, N. Sadati, and J. M. Guerrero, "Distributed noise-resilient secondary voltage and frequency control for islanded microgrids," IEEE Transactions on Smart Grid, vol. 10, no. 4, pp. 3780-3790, 2019.
[20] H.-M. Kim, T. Kinoshita, and M.-C. Shin, "A multiagent system for autonomous operation of islanded microgrids based on a power market environment," Energies, vol. 3, no. 12, pp. 1972-1990, 2010.

[21] R. Leo, A. A. Morais, R. Rathnakumar et al., "Micro-grid grid outage management using multi agent systems," in Proceedings of the 2 nd International Conference on Recent Trends and Challenges in Computational Models, Tindivanam, India, February 2017.

[22] A. Kantamnenia, L. E. Browna, G. Parkerb et al., "Survey of multi-agent systems for microgrid control," Engineering Applications of Artificial Intelligence, vol. 44, 2015.

[23] C. Karavas, K. Arvanitis, and G. Papadakis, "A game theory approach to multi-agent decentralized energy management of autonomous polygeneration microgrids," 2017.

[24] E. Rokrok, M. Shafie-khan, P. Siano, and J. P. S. Catalao, “A decentralized multi-agent based approach for low voltage microgrid restoration," Energies, vol. 22, 2017.

[25] R. R. Negenborn, B. De Schutter, and H. Hellendoorn, "Multiagent model predictive control of transportation networks," in Proceedings of the 2006 IEEE International Conference on Networking, Sensing and Control (ICNSC 2006), pp. 296-301, Lauderdale, FL, USA, April 2006.

[26] R. Halvgaard, "Model predictive control for smart energy systems," Technical University of Denmark Department of Applied Mathematics and Computer Science, vol. 23, 2017.

[27] J. Ma, F. Yang, Z. Li, and S. Joe Qin, "A renewable energy integration application in a microgrid based on model predictive control," IEEE Power and Energy Society General Meeting, vol. 23, pp. 22-26, 2012.

[28] F. Blanco, A. Labella, D. Mestriner, and A. Rosini, "Model Predictive Control for Primary Regulation of Islanded Microgrid," in Proceedings of the 2018 IEEE International Conference on Environment and Electrical Engineering and 2018 IEEE Industrial and Commercial Power Systems Europe (EEEIC/IঊCPS Europe), IEEE, Denmark, Europe, pp. 1-6, June 2018.

[29] H. T. Nguyen, E.-K. Kim, I.-P. Kim, H. H. Choi, and J.-W. Jung, "Model predictive control with modulated optimal vector for a three-phase inverter with an LC filter," IEEE Transactions on Power Electronics, vol. 33, no. 3, pp. 26902703, 2018.

[30] Q. Shafiee, T. Dragicevic, F. Andrade, J. C. Vasquez, and J. M. Guerrero, "Distributed consensus-based control of multiple DC-microgrids clusters," in Proceedings of the 2014 40th Annual Conference of the IEEE Industrial Electronics Society IECON, Singapore, February 2015.

[31] S. Ahmed Fuad, "Consensus based distributed control in microgrid consensus based distributed control in micro-grid clusters clusters," Master Thesis, Michigan Technological University, Houghton, Michigan, 2017.

[32] F. Aghaee, N. M. Dehkordi, N. Bayati, and A. Hajizadeh, "Distributed control methods and impact of communication failure in ac microgrids: a comparative review," Electronics, vol. 8, no. 11, p. 1265, 2019. 